\title{
A review on electronic and optical properties of silicon nanowire and its different growth techniques
}

\author{
Mehedhi Hasan ${ }^{1}$, Md Fazlul Huq ${ }^{2 *}$ and Zahid Hasan Mahmood ${ }^{3}$
}

\begin{abstract}
Electronic and optical properties of Silicon Nanowire (SiNW) obtained from theoretical studies and experimental approaches have been reviewed. The diameter dependency of bandgap and effective mass of SiNW for various terminations have been presented. Optical absorption of SiNW and nanocone has been compared for different angle of incidences. SiNW shows greater absorption with large range of wavelength and higher range of angle of incidence. Reflectance of SiNW is less than $5 \%$ over majority of the spectrum from the UV to near IR region. Thereafter, a brief description of the different growth techniques of SiNW is given. The advantages and disadvantages of the different catalyst materials for SiNW growth are discussed at length. Furthermore, three thermodynamic aspects of SiNW growth via the vapor-liquid-solid mechanism are presented and discussed.
\end{abstract}

Keywords: Silicon Nanowires (SiNWs), Bandgap, Optical absorption, Reflectance, Chemical Vapour Deposition (CVD), Molecular Beam Epitaxy (MBE)

\section{Introduction}

Growth of Si whiskers was first reported by Wagner and Ellis as early as 1964 (Wagner \& Ellis 1964). Later Givargizov elucidated the growth mechanism of Si whiskers in 1975 (Givargizov 1975). The first report on carbon nanotubes by Iijima in 1991 focused a worldwide exponential increase of research into the carbon based and siliconbased nanomaterials especially carbon nanotubes and SiNWs (Iijima 1991). Subsequently, there were extensive investigations carried out on the synthesis, physical properties, device fabrication and applications of SiNWs. Figure 1 shows a histogram of the number of silicon "whisker" and "nanowire" publications (Schmidt \& Wittemann 2009). Due to their novel properties Silicon nanowires (SiNWs) have grabbed such great attentions. It is their unique electrical, optical and mechanical properties that make them to receive such interest. Even more importantly, SiNWsbased nanodevices are compatible with the current Si-based microelectronics industry, and already a number

\footnotetext{
* Correspondence: fhuq.apece.du@gmail.com

2Department of Information and Communication Technology, Mawlana Bhashani Science and Technology University, Santash, Tangail 1902, Bangladesh

Full list of author information is available at the end of the article
}

of nanodevices based on SiNWs as building blocks have been demonstrated (Cui \& Lieber 2001).

The nanoscale diameter puts the radial dimension of nanowires at or below the characteristic length scale of various interesting and fundamental solid state phenomena: the exciton Bohr radius, wavelength of light, phonon mean free path, critical size of magnetic domains, exciton diffusion length, etc. (Alivisatos 1996; Law et al. 2004). As a result, many physical properties of semiconductors are significantly altered within the confinement of the nanowire surfaces. In addition, their large surfaceto-volume ratio allows for distinct structural and chemical behavior as well as greater chemical reactivity. This two-dimensional confinement endows nanowires with unique properties which stray from those of their corresponding bulk material. Second, the large aspect ratio of nanowires intimates their technological application. The one unconstrained dimension can direct the conduction of quantum particles such as electrons, phonons, and photons. This control over various forms of energy transport recommends nanowires as ideal materials from which to manufacture advanced solid-state devices. Moreover, the length of nanowires is normally sufficient to interface with top-down fabrication processes, such as 


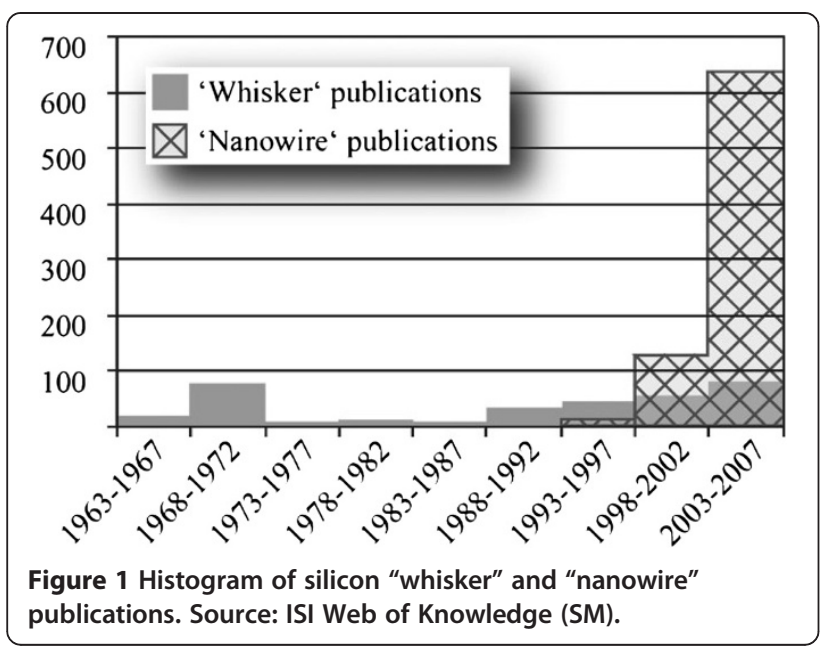

photolithography. As a result, nanowires provide a convenient platform through which researchers may study confined transport phenomena.

\section{Review \\ Properties of Silicon Nanowires Electronic Properties}

The small sizes of SiNWs make their electronic and electrical properties strongly dependent on growth direction, size, morphology and surface reconstruction. A well known example is the size dependence of the electronic bandgap width of SiNWs irrespective of wire direction. As the wire diameter decreases, the band gap of the nanowire widens and deviates from that of bulk silicon gradually. Moreover, the orientations of the wire axis and the surface have a great effect on the electronic properties of SiNWs (Aijiang 2007).

Michael Nolan et al. theoretically investigated the band gap modification for small-diameter $(\sim 1 \mathrm{~nm})$ silicon nanowires resulting from the use of different species for surface termination by density functional theory calculations. Because of quantum confinement, small-diameter wires exhibit a direct band gap that increases as the wire diameter narrows, irrespective of surface termination. Figure 2 shows band gap as a function of nanowire diameter for various surface terminations (Nolan et al. 2007).

Sacconi et al. also investigated the electronic properties of silicon nanowires with several different approaches like the Empirical Tight-Binding (ETB) model, the Linear Combination of Bulk Bands (LCBB) model and NonEquilibrium Green Function (NEGF) model. They considered both hydrogenated and $\mathrm{SiO}_{2}$ terminated silicon surfaces in these models. When the diameter of SiNW is reduced from 3.2 to $1.6 \mathrm{~nm}$, the bandgap of hydrogenated nanowire increases from 1.56 to $2.44 \mathrm{eV}$. On the other hand, in the case of $\mathrm{SiO}_{2} / \mathrm{SiNW}$ structure, this increase is smaller, since the bandgap goes from $1.50 \mathrm{eV}$ for a $3.2 \mathrm{~nm}$ cell, to $1.88 \mathrm{eV}$ for a $1.6 \mathrm{~nm}$ cell. This behavior can be expected due to a lower confinement determined by $\mathrm{SiO}_{2}$ surrounding SiNW, with respect to the case of simple hydrogen termination.

Effective masses for conduction and valence bands have also been calculated. The effect of an increasing $\mathrm{Si}$ thickness on a hydrogen terminated wire is that of reducing the conduction mass, from $0.47 \mathrm{~m}_{\mathrm{o}}$ to $0.31 \mathrm{~m}_{\mathrm{o}}$, which is $55 \%$ greater than the value of transverse mass in bulk silicon. The effect on the $\mathrm{SiO}_{2}$-confined wire is similar; by increasing $\mathrm{Si}$ thickness, effective mass decreases from $0.36 \mathrm{~m}_{\mathrm{o}}$ to $0.29 \mathrm{~m}_{\mathrm{o}}$ (Sacconi et al. 2007).

The effect of wire thickness of SiNW on conduction valley splitting, hole band splitting, effective masses and transmission has been reported using $\mathrm{sp}^{3} \mathrm{~d}^{5} \mathrm{~s}^{*}$ model by Yun Zheng et al. They concluded that in the conduction band, valley splitting reduces the averaged mobility mass along the axis of the wire, but quantum confinement increases the transverse mass of the conduction band edge. For the wire thickness range that they have considered, the effective mass at the conduction band edge is at least $35 \%$ heavier than that of transverse mass of bulk Si. Quantum confinement has the largest effect on the effective masses in the valence band. The effective mass at the valence band edge is at least six times heavier than that of the bulk. The effective mass of the next highest band is even heavier. Small energy splitting also occurs at the conduction band minimum. For wires greater than $1.54 \mathrm{~nm}$ thick, the four bulk valleys which compose the conduction band minimum are split into three energies. The center energy is twofold degenerate roughly evenly split between the lowest and highest energy. The single-band model performs reasonably well at calculating the effective band edges for the $1.54 \mathrm{~nm}$ wire (Zheng et al. 2005).

Yi Cui et al. shows that Boron and phosphorus can be used to change the conductivity of SiNWs over many orders of magnitude and that the conductivity of the doped SiNWs respond oppositely to positive (negative) $V \mathrm{~g}$ for boron and phosphorus dopants. Indeed, the $V \mathrm{~g}$ dependence provides strong proof for p-type (holes) doping with boron and n-type (electrons) doping with phosphorus in the SiNWs (Cui et al. 2000).

\section{Optical Properties}

Bulk Si has an indirect band gap, with the valence band maximum at the $\Gamma$ point and the conduction minimum at about $85 \%$ along the $\Gamma$ to $\mathrm{X}$ direction, and a phonon is required to conserve the momentum in any electronic transition. Remarkably, however, SiNWs grown along most of the crystallographic orientations have a direct band gap, meaning that the maximum of the valence band and the minimum of the conduction band occur at the same point in k-space. This property has allowed to envisage the use 

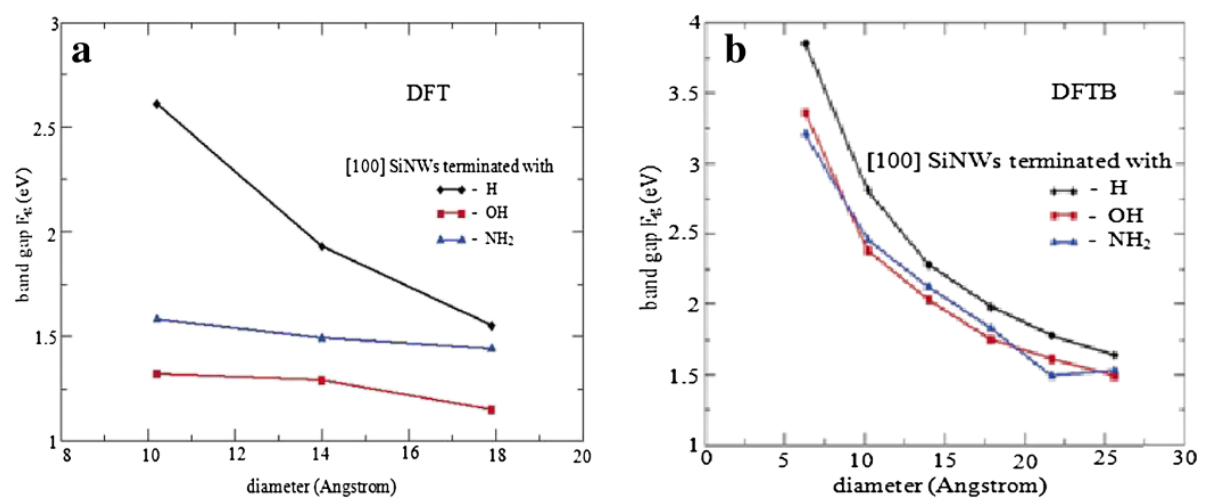

Figure 2 Band gap as a function of the [100] silicon nanowire diameter for various surface terminations. (a) DFT calculations within GGAPBE. (b) Results from a density-functional tightbinding (DFTB) parameterization.

of SiNWs as optically active materials for photonics applications (Canham 1990; Guichard et al. 2006).

The possibility of controlling the band gap width is tremendously attractive for optoelectronics applications: not only SiNWs can have a direct band gap, which per se increases the optical efficiency, but its width can in principle be tuned. It is not difficult to imagine, however, that controlling the wire diameter with tolerances within $1-3 \mathrm{~nm}$ is a more than challenging task. A simpler route to band gap tuning is controlling the chemical composition and the coverage density of the wire surface. Halogens such as $\mathrm{Cl}, \mathrm{Br}$, and I can be used as surface passivation agents instead of $\mathrm{H}$ and, while not altering the semiconducting character of the wires, they result in a significant shrinking of band gap (Leu et al. 2006). The strongest reduction of the band gap is provided by $\mathrm{I}$, followed by $\mathrm{Br}$ and $\mathrm{Cl}$, in the opposite order of the bonding strength of these species and SiNWs. Interestingly, the surface coverage is a further degree of freedom and one can span all the band gap values between a $\mathrm{H}$ - and halogen-passivated wire by varying the $\mathrm{H}$ : halogen ratio. Also, increasing the halogen surface concentration the band edge states, concentrated in the wire core in presence of $\mathrm{H}$-passivation, progressively spread to the surface.

Analogous results have been reported for $\mathrm{OH}$ and $\mathrm{NH} 2$ (Aradi et al. 2007; Nolan et al. 2007). It should be noted that the passivating species do not contribute significantly to the states close to the band edges, so that the reduction of the gap is not caused by the introduction of additional bands. It rather comes from the hybridization of the valence band states with the frontier orbitals of the different passivating functional groups that cause a significant band gap reduction relative to $\mathrm{H}$-passivated wires.

These results indicate that the band gap width in SiNWs can be tailored not only by controlling the wire diameter, but also by an appropriate choice of the surface termination.
The broadband optical absorption properties of silicon nanowire (SiNW) films have been measured and found to be higher than that of solid thin films of Si of equivalent thickness. The observed behavior is adequately explained by light scattering and light trapping though some of the observed absorption is due to a high density of surface states in the nanowires films, as evidenced by the partial reduction in high residual subbandgap absorption after hydrogen passivation. The reflectance of the solid film shows typical behavior what expected for silicon, whereas the reflectance of the nanowire film is less than $5 \%$ over the majority of the spectrum from the UV to the near IR and begins to increase at $\sim 700 \mathrm{~nm}$ to a values of $\sim 41 \%$ at the Si band edge $(1100 \mathrm{~nm})$, similar to the solid film sample. It is clear that the nanowires impart a significant reduction of the reflectance compared to the solid film. Figure 3 shows comparative reflectance of solid silicon and $\mathrm{Si}$ nanowires (Tsakalakos et al. 2007).

Fabrication of $\mathrm{Si}: \mathrm{H}$ nanowires (NWs) and nanocones (NCs), using an easily scalable and IC-compatible process

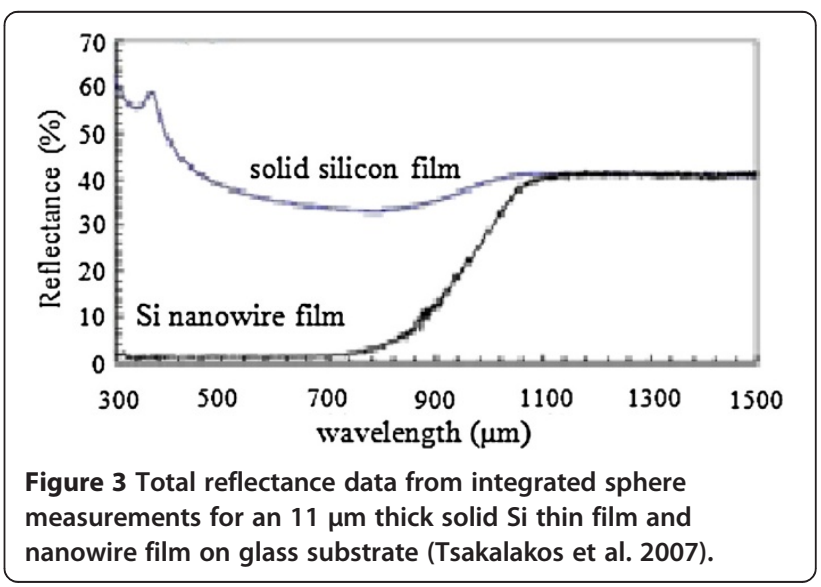


has been reported by Jia Zhu et al. They have shown that Si:H nanostructures display greatly enhanced absorption over a large range of wavelengths and angles of incidence due to suppressed reflection. More than $90 \%$ of light is absorbed at angles of incidence up to $60^{\circ}$ for a-Si:H NC arrays, which is significantly better than NW arrays (70\%) and thin films (45\%). In addition, the absorption of NC arrays is $88 \%$ at the band gap edge of a- $\mathrm{Si}: \mathrm{H}$, which is much higher than NW arrays (70\%) and thin films (53\%). Figure 4 summarized the results of different structures for different condition (Zhu et al. 2009).

\section{Methods of Fabrication of Nanowires}

Many techniques, including both top-down and bottom-up approaches, have been developed and applied for the synthesis of Nanowires. Vapor-Liquid-Solid (VLS) Mechanism, Chemical Vapor Deposition (CVD), Evaporation of SiO,
Molecular Beam Epitaxy (MBE), Laser Ablation and Electroless metal deposition and dissolution (EMD) have been discussed here.

\section{Vapor-Liquid-Solid (VLS) Mechanism}

The VLS mechanism, first proposed by Wagner and Ellis (Wagner \& Ellis 1964) in the mid-1960s, is the key mechanism for silicon-wire growth. Their proposed VLS mechanism is based on two observations: that the addition of certain metal impurities is an essential prerequisite for growth of silicon nanowires in experiments and that small globules of the impurity are located at the tip of the wire during growth. From this, Wagner and Ellis deduced that the globule at the wire tip must be involved in the growth of the silicon wires by acting "as a preferred sink for the arriving $\mathrm{Si}$ atoms or, perhaps more likely, as a catalyst for the chemical process involved"
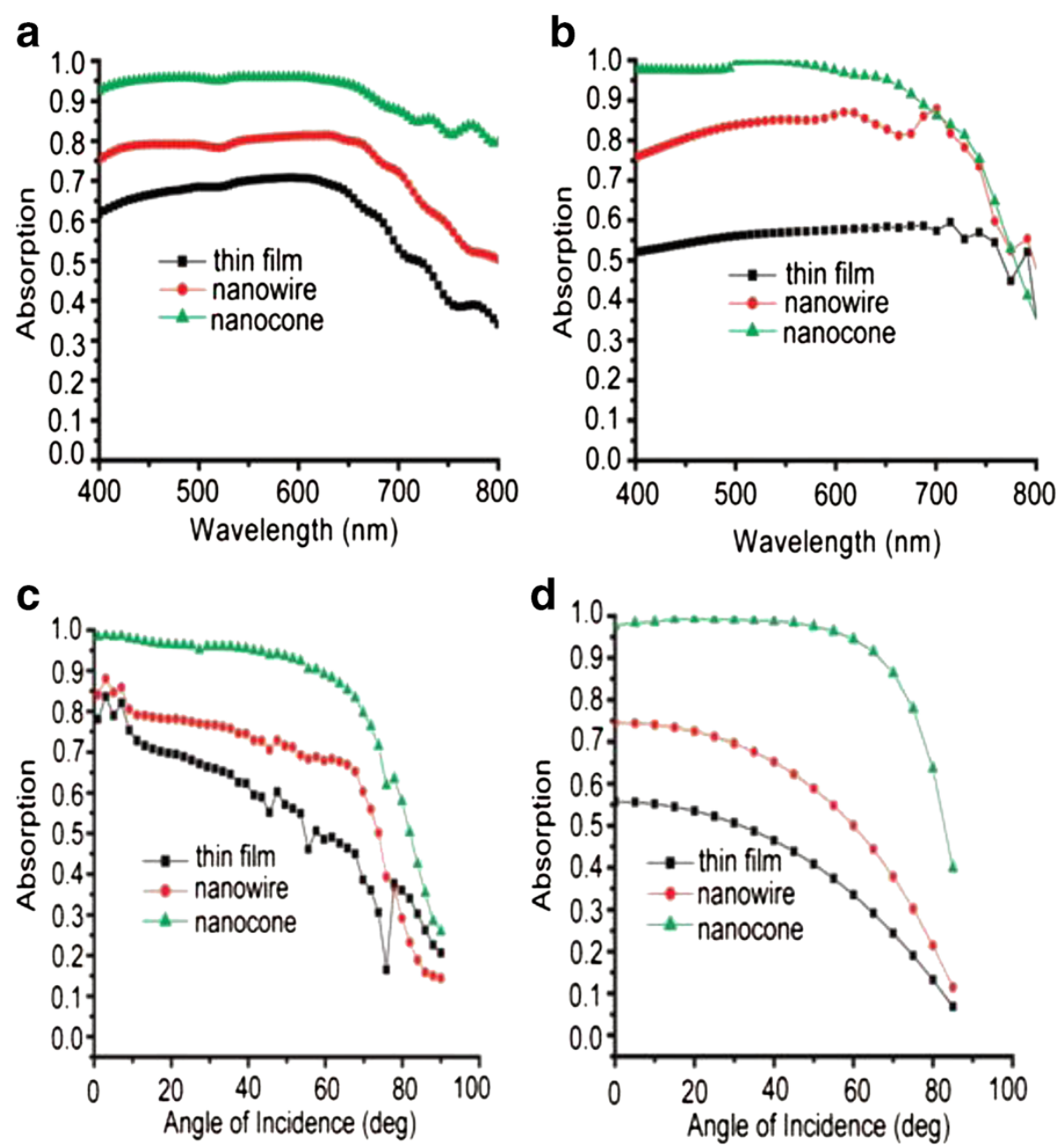

Figure 4 Value of absorption on samples with a-Si:H thin film, NW arrays and NC arrays (a) Measured, (b) Calculated over a large range of wavelengths at normal incidence; (c) Measured (d) Simulated for different angle of incidence (at wavelength $\lambda=488 \mathrm{~nm}$ ) (Zhu et al. 2009). 
(Wagner \& Ellis 1964). When Au, for example, is deposited on silicon substrate and this substrate is then heated to temperatures above about $363^{\circ} \mathrm{C}$, small liquid $\mathrm{Au}-\mathrm{Si}$ alloy droplets will form on the substrate surface. Exposing such a substrate to a gaseous silicon precursor, such as silicon tetrachloride $\left(\mathrm{SiCl}_{4}\right)$ or silane $\left(\mathrm{SiH}_{4}\right)$ precursor molecules will crack on the surface of the $\mathrm{Au}-\mathrm{Si}$ alloy droplets, whereupon $\mathrm{Si}$ is incorporated into the droplet. The silicon supply from the gas phase causes the droplet to become supersaturated with $\mathrm{Si}$ until silicon freezes out at the silicon/ droplet interface. The continuation of this process then leads to the growth of a wire with the alloy droplet riding atop the growing wire (Wagner \& Ellis 1964) (Figure 5).

\section{Chemical Vapor Deposition (CVD)}

In CVD, a volatile gaseous silicon precursor, such as silane $\left(\mathrm{SiH}_{4}\right)$ or silicon tetrachloride $\left(\mathrm{SiCl}_{4}\right)$, serves as the silicon source. It is transported to the deposition surface at which the precursor reacts, and is cracked into its constituents as depicted in Figure 6.

Originally, CVD was devised for the deposition of high-purity films. Contaminations such as gold particles, however, were found to cause anisotropic growth of silicon, that is, the growth of silicon wires. CVD allows epitaxial growth of SiNWs, with the growth velocity varying from about $10^{-2}$ to $10^{3} \mathrm{~nm} \mathrm{m^{-1 }}$, (Kodambaka et al. 2006; Nebol'sin et al. 2005) depending on temperature and type of Si precursor used. Furthermore, CVD offers broad possibility of modifying the properties of the silicon wires in a controlled fashion. A variety of derivatives of CVD methods exist. These can be classified by parameters such as the base and operation pressure or the treatment of the precursor. Since silicon is known to oxidize easily if exposed to oxygen at elevated temperatures, it is crucial to reduce the oxygen background pressure in order to be able to epitaxially grow uniform

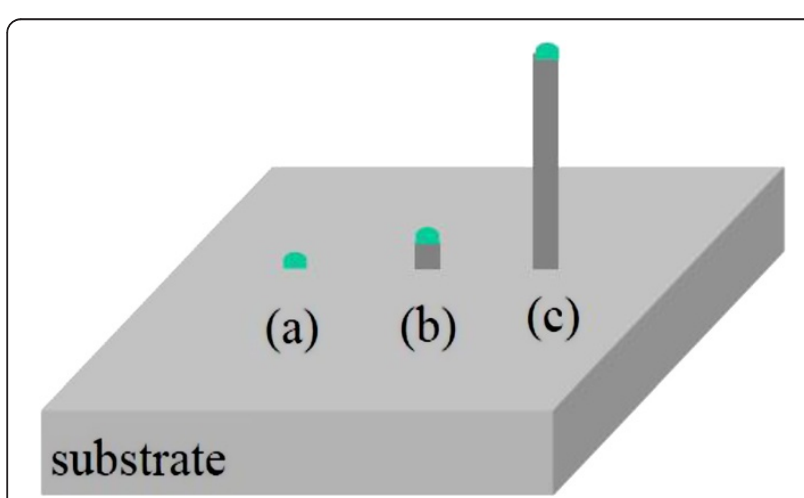

Figure 5 Schematic of the VLS growth mechanism (a) Catalytic liquid alloy $(b, c)$ Successive growth of nanowire.

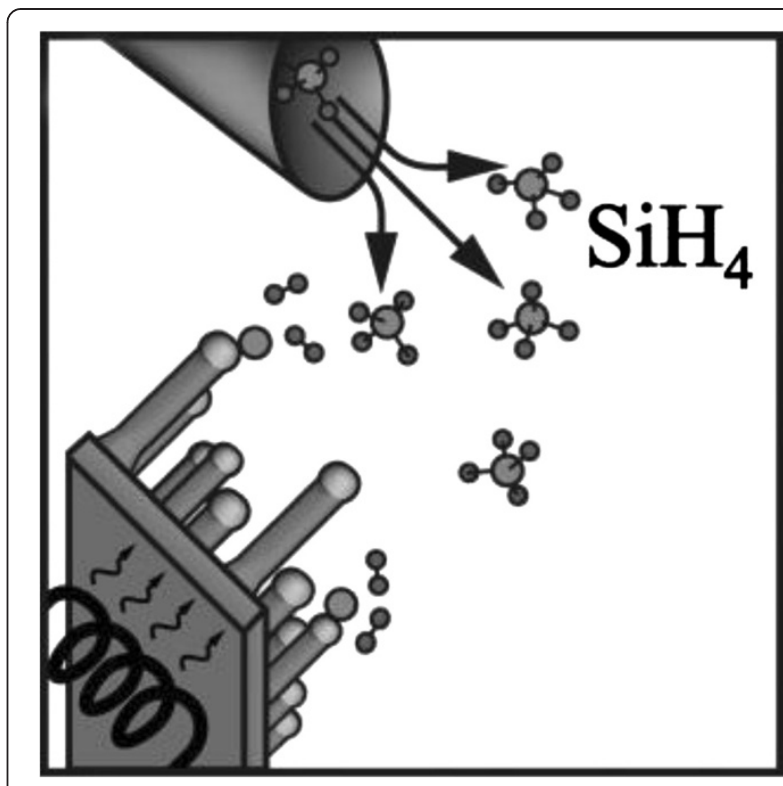

Figure 6 Schematics of experimental setup for nanowire growth using CVD method.

silicon nanowires. In particular, when oxygen-sensitive catalyst materials are used, it turns out to be useful to combine catalyst deposition and nanowire growth in one system, so that growth experiments can be performed without breaking the vacuum in between (Wang et al. 2006). In any case, it is useful to lower the base pressure of the CVD reactor down to high or even ultrahigh vacuum, which reduces unwanted contamination and enables growth at lowered temperatures (Akhtar et al. 2008). The pressures during growth mainly depends upon the gaseous silicon precursor and its cracking probability at the catalyst surface. Growth with disilane, $\mathrm{Si}_{2} \mathrm{H}_{6}$, for example, can-but must not-be carried out at extremely low partial pressures of around $10^{-6} \mathrm{mbar}$ ( 1 bar $=10^{5} \mathrm{~Pa}$ ). These low growth pressures allow the combination of CVD with transmission electron microscopy (TEM), enabling in situ observation of the nanowire growth (Hofmann et al. 2008). In contrast to that, silane partial pressures required for wire growth are about five orders of magnitude higher. By modifying the precursor before reacting with the sample surface, the temperature budget of the substrate can be lowered. In cases where the thermal load is critical or where a high supersaturation of the droplet is necessary, nanowire growth can be enhanced using plasma-enhanced CVD (PECVD) (Hofmann et al. 2003; Sharma et al. 2004; Iacopi et al. 2007). Another advantage of CVD as a bottom-up synthesis method is its variability concerning the intended wire size. Wire diameters range from below $10 \mathrm{~nm}$ (Cui et al. 2001) up to several hundred micrometers (Wagner \& Ellis 1964). Since surface diffusion only plays a minor role in CVD, the 
length of the wires can also be tuned accordingly by simply extending or decreasing the growth time. Thus, to summarize, a large range of length and diameter configurations can be fabricated (Park et al. 2008). With CVD, not only the wire size but also its properties can be modified.

\section{Evaporation of SiO}

A cost-effective method to produce silicon nanowires on a large scale is to evaporate solid silicon monoxide, $\mathrm{SiO}$ (shown in Figure 7).

A two-zone tube furnace connected to an inert gas supply and small amount of $\mathrm{SiO}$ granulate are the basic ingredients for the synthesis of silicon nanowires. Crucial for growth is a temperature gradient from about 1350 to $900^{\circ} \mathrm{C}$ along the tube of the furnace. $\mathrm{SiO}$ is evaporated at the hotter end of the tube, flows with the gas stream to the cooler part, where it undergoes a disproportionation reaction into $\mathrm{Si}$ and $\mathrm{SiO}_{2}$, thereby forming the nanowires (Pan et al. 2001). In principle, two different growth methods are possible: growth with and without metal catalyst. Growth assisted by the presence of a metal catalyst is relatively rapid (Gu et al. 2000). Consistent with the concept of VLS growth, the diameters are determined by the size of the catalyst particle, although the interplay between the nanowire and the catalyst droplet seems to be more complex compared to normal CVD growth. As a consequence of the disproportionation reaction, the diameter ratio between crystalline core and amorphous shell remains approximately constant (Kolb et al. 2004). The second growth mode,

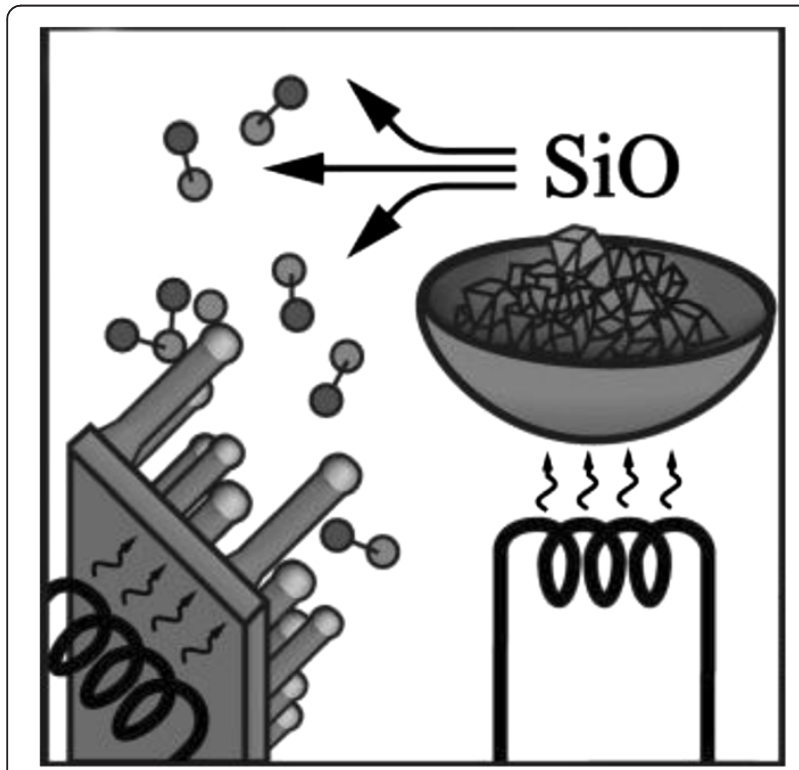

Figure 7 Schematics of experimental setup for silicon nanowire growth by evaporation of SiO. metal-catalyst-free growth, has been originally proposed for growth via laser ablation (Wang et al. 1998), where it was observed that nanowires can be catalyzed by silicon dioxide (Wang et al. 1999). Remarkable about this oxide-assisted growth (OAG) is that $\mathrm{SiO}_{2}$-containing targets clearly raise the yield of the final amount of silicon nanowires compared to pure silicon targets or mixed silicon-metal targets (Wang et al. 1998). By carrying out the growth process over several hours, one can obtain millimeter-long crystalline silicon nanowires with varying diameters from about $5 \mathrm{~nm}$ to $100 \mathrm{~nm}$, covered by an amorphous shell of up to several $10 \mathrm{~nm}$ (Shi et al. 2000; Zhang et al. 2000; Shi et al. 2005).

\section{Molecular Beam Epitaxy (MBE)}

In MBE, a solid high-purity silicon source is heated until Si starts to evaporate. Figure 8 schematically depicts an MBE setup.

A directional gaseous beam of silicon atoms is aimed at the substrate, on which the atoms adsorb and crystallize. To reduce contamination, the base pressure of an MBE system is usually kept at ultrahigh vacuum, allowing to monitor the growth using Reflection High-Energy Electron Diffraction (RHEED) (Werner et al. 2006) or other surface sensitive examination methods. Similar to CVD, MBE was initially designed for epitaxial layer-by-layer deposition only. Yet, metal contamination was also found to cause silicon-wire growth in this case. Differing from CVD, no precursor gas is cracked at the surface of the liquid metal-silicon alloy. Therefore, the latter cannot be treated as a classical catalyst anymore. In MBE, two silicon fluxes govern wire growth. First, the direct flux of silicon from the silicon source; and second, the flux of diffusing

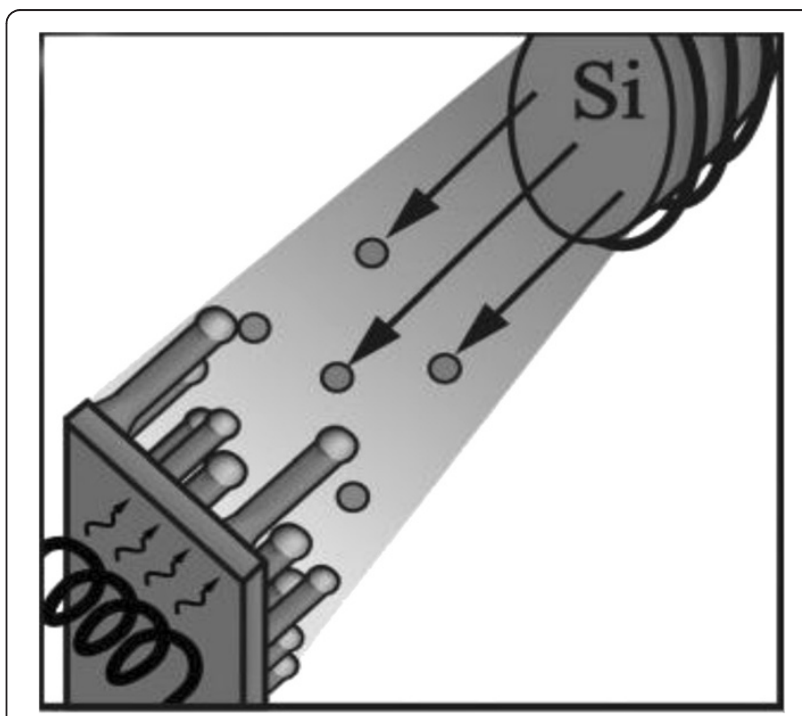

Figure $\mathbf{8}$ Schematics of experimental setup for silicon nanowire growth by MBE. 
silicon adatoms from the silicon substrate surface. The nanowires produced by $\mathrm{MBE}$ - usually grown on $\mathrm{Si}(111)$ substrates-are epitaxial and $<111>$ oriented. MBE offers excellent controllability in terms of the incoming flux, such that doped wires (Kanungo et al. 2008) or heterostructures (Zakharov et al. 2006) can be grown by switching between evaporation sources. One disadvantage of MBE, however, is that the method is limited with respect to the minimally possible Si-nanowire diameter. Only nanowires with diameters greater than about $40 \mathrm{~nm}$ can be obtained (Shi et al. 2005; Schubert et al. 2004) which seems to be a consequence of the Gibbs-Thomson effect and the fact that only small Si supersaturations are achievable by MBE. Another disadvantage of $\mathrm{MBE}$ is the low nanowire growth velocity of just a few nanometers per minute (Schubert et al. 2004).

\section{Laser Ablation}

The silicon nanowires produced by laser ablation differ in many aspects from the MBE grown whiskers. One can easily obtain large quantities of ultrathin nanowires with high aspect ratios (Zhang et al. 1998; Zhou et al. 1998). As schematically displayed in Figure 9, a high-power pulsed laser ablates material from a mixed $\mathrm{Si}$-catalyst target, which is placed in a tube furnace held at high temperatures and purged with an inert gas.

The silicon material ablated from the target cools by colliding with inert-gas molecules, and the atoms condense to liquid nanodroplets with the same composition as the target (Morales \& Lieber 1998). Thus, these nanoparticles contain both $\mathrm{Si}$ and the catalyst material. According to the VLS mechanism, silicon nanowires start to grow once the

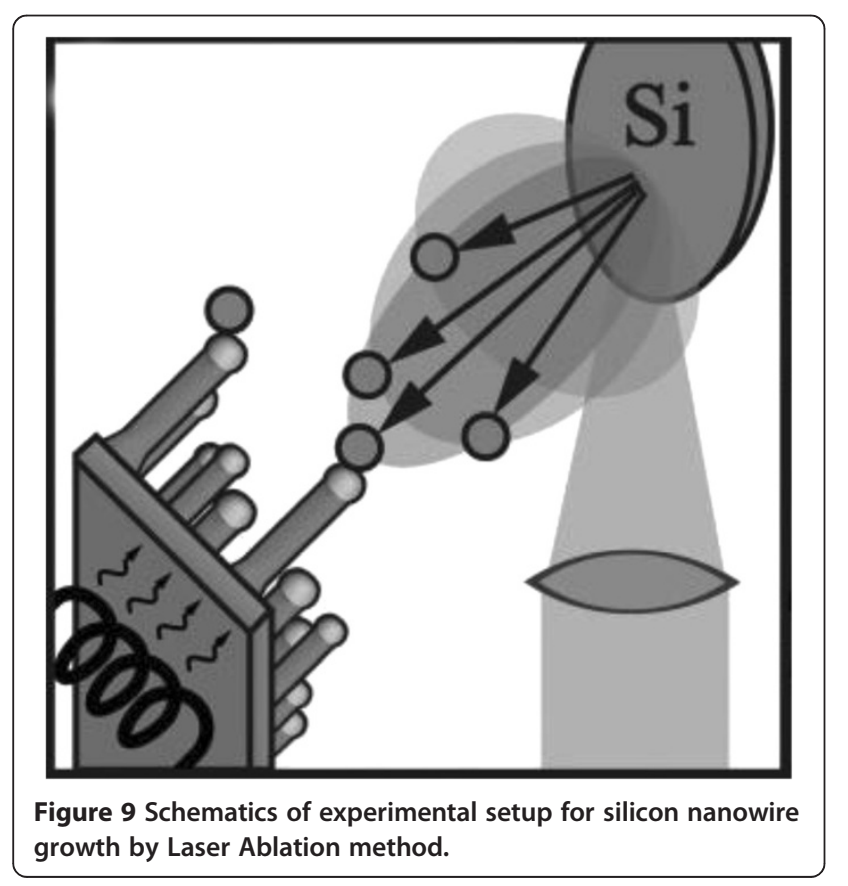

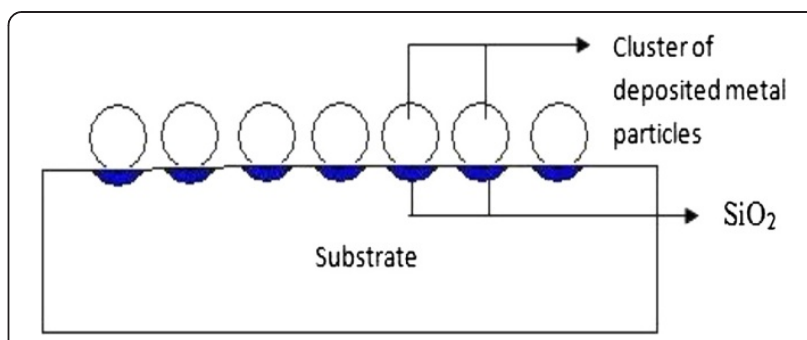

Figure 10 Oxidation of silicon surface under deposited metal.

catalyst gets supersaturated with silicon and proceeds as long as the catalyst nanoparticles remain liquid. The advantages of laser-ablated nanowire production are manifold. First, there is no need for a substrate. Second, the composition of the resulting nanowires can be varied by changing the composition of the laser target. By adding, for example, $\mathrm{SiO}_{2}$ to the target, single-crystalline silicon nanowires with varied amorphous $\mathrm{SiO}_{\mathrm{x}}$ shell thicknesses can be obtained in a single processing step (yang et al. 2004) with silicon-core diameters as low as $5 \mathrm{~nm}$ and varying shell thicknesses of about $10 \mathrm{~nm}$. Due to the high growth temperatures, catalyst metals such as Fe, possessing a high eutectic temperature, can be used. The resulting nanowire growth velocities are typically of the order of micrometers per minute (Zhang et al. 1998; Morales \& Lieber 1998). The radii of the nanowires not only depend on the type of metal catalysts used but also on the gases that are streamed through the furnace, such as $\mathrm{H}_{2}, \mathrm{He}$, or $\mathrm{N}_{2}$ (Zhang et al. 1999).

\section{Electroless metal deposition and dissolution}

Electroless deposition, a non-galvanic type of deposition method that involves several simultaneous reactions in an aqueous solution, which occur without the use of external electrical power. The most common electroless deposition is nickel, silver or gold particle deposition. First step of silicon nanowire synthesis using this process is to deposit metal particle like $\mathrm{Au}, \mathrm{Ag}$ or $\mathrm{Cu}$ on silicon substrate. These noble metals would attract electrons from the silicon and facilitate Si oxidation. Figure 10 shows the oxidation of silicon surface under deposited metal.

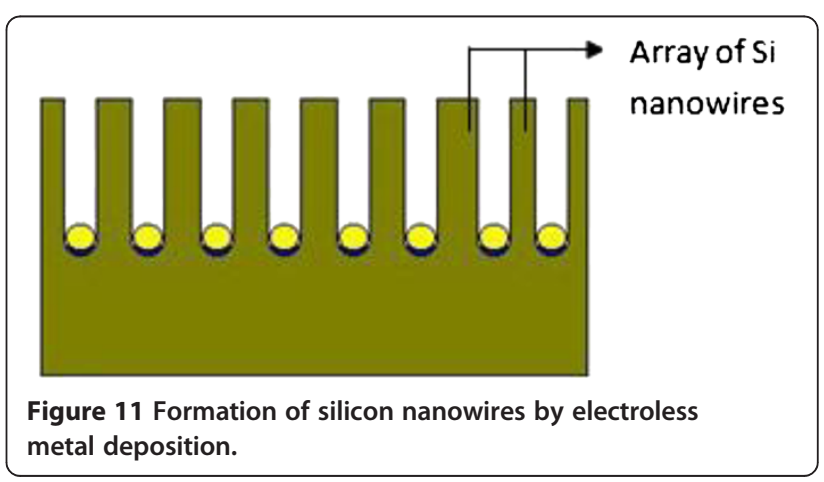


Generally, the etching of a cleaned $\mathrm{Si}$ wafer proceeds very slowly in aqueous $\mathrm{HF} / \mathrm{Fe}\left(\mathrm{NO}_{3}\right)_{3}$ solution at low temperature. However, Si etching occurs rapidly when $\mathrm{Si}$ substrates covered with $\mathrm{Ag} / \mathrm{Au}$-nanoparticle films are immersed in $\mathrm{HF} / \mathrm{Fe}\left(\mathrm{NO}_{3}\right)_{3}$ solution at room temperature. Formation of SiNW arrays due to the further sinking of the Ag particles, and longitudinal and lateral dissolution of bulk Si (shown in Figure 11) (Peng et al. 2006).

\section{Conclusions}

In summary, we have seen that there are significant changes in electronic and optical properties of silicon nanowires than those of bulk. The change of some properties depends on the size and shape of the nanostructures. It suggests that, careful production of nanowires of desired size and shape would make it possible to manipulate properties like bandgap, effective mass and optical absorption. It also has been seen that silicon nanowires can be produced by different growth methods. Au and Ag are the most popular catalyst material for silicon nanowire synthesis.

\section{Competing interest}

The author declares that they have no competing interest.

\section{Authors' contributions}

All authors have equal contribution on this work and all authors read and approved the final manuscript.

\section{Author details \\ 'Department of Electrical and Electronic Engineering, Shahjalal University of Science and Technology, Kumargaon, Sylhet 3114, Bangladesh. ${ }^{2}$ Department of Information and Communication Technology, Mawlana Bhashani Science and Technology University, Santash, Tangail 1902, Bangladesh. ${ }^{3}$ Department of applied Physics Electronics and Communication Engineering, University of Dhaka, Dhaka 1000, Bangladesh.}

Received: 15 September 2012 Accepted: 1 April 2013

Published: 10 April 2013

\section{References}

Aijiang LU (2007) Theoretical Study of Electronic and Electrical Properties of Silicon Nanowires. Dissertation, City University of Hong Kong, Hong Kong

Akhtar S, Usami K, Tsuchiya Y, Mizuta H, Oda S (2008) Vapor-Liquid-Solid Growth of Small- and Uniform-Diameter Silicon Nanowires at Low Temperature from $\mathrm{Si}_{2} \mathrm{H}_{6}$. Appl Phys Express 1:014003-5

Alivisatos AP (1996) Semiconductor Clusters, Nanocrystals, and Quantum Dots. Science 271(5251):933-937

Aradi B, Ramos LE, De'ak P, K"ohler T, Bechstedt F, Zhang RQ, Frauenheim T (2007) Theoretical study of the chemical gap tuning in silicon nanowires. Phys Rev B 76(3):1-7, 035305

Canham LT (1990) Silicon quantum wire array fabrication by electrochemical and chemical dissolution of wafers. Appl Phys Lett 57(10):1046-1048

Cui Y, Duan X, Hu J, Lieber CM (2000) Doping and Electrical Transport in Silicon Nanowires. J Phys Chem B 104(22):5213-5216

Cui Y, Lauhon L, Gudiksen MS, Wang J, Lieber CM (2001) Diameter-controlled synthesis of single-crystal silicon nanowires. Appl Phys Lett 78:2214-2216

Cui Y, Lieber CM (2001) Assembled using Silicon Nanowire Building Blocks Functional Nanoscale Electronic Devices. Science 291:851-853

Givargizov El (1975) Fundamental Aspects of VLS Growth. J. Cryst Growth 31:20-30

Guichard AR, Barsic DN, Sharma S, Kamins TI, Brongersma ML (2006) Tunable light emission from quantum-confined excitons in TiSi2-catalyzed silicon nanowires. Nano Lett 6(9):2140-4

Gu Q, Dang H, Cao J, Zhao J, Fan S (2000) Silicon nanowires grown on ironpatterned silicon substrates. Appl Phys Lett 76:3020-3021
Hofmann S, Ducati C, Neill RJ, Piscanec S, Ferrari AC, Geng J, Dunin-Borkowski RE, Robertson J (2003) Gold catalyzed growth of silicon nanowires by plasma enhanced chemical vapor deposition. J Appl Phys 94(9):6005-6012

Hofmann S, Sharma R, Wirth CT, Cervantes-Sodi F, Ducati C, Kasama T, DuninBorkowski RE, Drucker J, Bennett P, Robertson J (2008) Ledge-flow-controlled catalyst interface dynamics during Si nanowire growth. Nat Mater 7:372-375

lacopi F, Vereecken PM, Schaekers M, Caymax M, Moelans N, Blanpain B, Richard O, Detavernier C, Griffiths H (2007) Plasma-enhanced chemical vapour deposition growth of Si nanowires with low melting point metal catalysts: an effective alternative to Au-mediated growth. Nanotechnology 18:1-7, 505307

lijima S (1991) Helical Microtubules of Graphitic Carbon. Nature 354:56-58

Kanungo PD, Zakharov N, Bauer J, Breitenstein O, Werner P, Goesele U (2008) Controlled in situ boron doping of short silicon nanowires grown by molecular beam epitaxy. Appl Phys Lett 92:263107-9

Kodambaka S, Tersoff J, Reuter MC, Ross FM (2006) Diameter-Independent Kinetics in the Vapor-liquid-Solid Growth of Si Nanowires. Phys Rev Lett 96 (9):096101-5

Kolb FM, Hofmeister H, Scholz R, Zacharias M, Go"sele U, Ma DD, Lee ST (2004) Analysis of silicon nanowires grown by combining $\mathrm{SiO}$ evaporation with the VLS mechanism. J Electrochem Soc 151:G472-G475

Law M, Goldberger J, Yang PD (2004) Semiconductor Nanowires and Nanotubes Annu. ReV Mater Res 34:83-122

Leu PW, Shan B, Cho K (2006) Surface chemical control of the electronic structure of silicon nanowires: Density functional calculations. Phys Rev B 73 (19):195320-4

Morales AM, Lieber CM (1998) A Laser Ablation Method for the Synthesis of Crystalline Semiconductor Nanowires. Science 279(5348):208-211

Nolan M, O'Callaghan S, Fagas G, Greer JC, Frauenheim T (2007) Silicon nanowire band gap modification. Nano Lett 7(1):34-38

Nebol'sin VA, Shchetinin AA, Dolgachev AA, Korneeva W (2005) Effect of the Nature of the Metal Solvent on the Vapor-liquid-Solid Growth Rate of Silicon Whiskers Inorg. Mater 41(12):1256-1259

Pan ZW, Dai ZR, Xu L, Lee ST, Wang ZL (2001) Temperature controlled growth of silicon-based nanostructures by thermal evaporation of SiO powders. J Phys Chem B 105:2507-2514

Park WI, Zheng G, Jiang X, Tian B, Lieber CM (2008) Controlled Synthesis of Millimeter-Long Silicon Nanowires with Uniform Electronic Properties. Nano Lett 8(9):3004-3009

Peng KQ, Hu JJ, Yan YJ, Wu Y, Fang H, Xu Y, Lee ST, Zhu J (2006) Fabrication of Single-Crystalline Silicon Nanowires by Scratching a Silicon Surface with Catalytic Metal Particles. Adv Funct Mater 16(3):387-394

Sacconi F, Persson MP, Povolotskyi M, Latessa L, Pecchia A, Gagliardi A, Balint A, Frqunheim T, Carlo AD (2007) Electronic and transport properties of silicon nanowires. J Comput Electron 6:329-333

Schmidt V, Wittemann JV (2009) Silicon Nanowires: A Review on Aspects of their Growth and their Electrical Properties. Adv Mater 21:2681-2702

Schubert L, Werner P, Zakharov ND, Gerth G, Kolb FM, Long L, Go" sele U, Tan TY (2004) Silicon nanowhiskers grown on $\langle 111\rangle$ Si substrates by molecular-beam epitaxy. Appl Phys Lett 84:4968-4970

Sharma S, Sunkara MK (2004) Direct synthesis of single-crystalline silicon nanowires using molten gallium and silane plasma. Nanotechnology 15 (1):130-134

Shi WS, Peng HY, Zheng YF, Wang N, Shang NG, Pan ZW, Lee CS, Lee ST (2000) Synthesis of Large Areas of Highly Oriented. Very Long Silicon Nanowires. Adv Mater 12(18):1343-1345

Shi Y, Hu Q, Araki H, Suzuki H, Gao H, Yang W, Noda T (2005) Long Si nanowires with millimeter-scale length by modified thermal evaporation from Si powder. Appl Phys A 80(8):1733-1736

Tsakalakos L, Balch J, Fronheiser J, Shih M, LeBoeuf SF, Pietrzykowski M, Codella PJ, Korevaar BA, Sulima OV, Rand J, Davuluru A, Rapol U (2007) Strong broadband optical absorption in silicon nanowire films. Journal of Nanophotonics 1(1):1-10, 013552

Wagner RS, Ellis WC (1964) Vapor-liquid-Solid Mechanism of Single Crystal Growth. Appl Phys Lett 4:89-90

Wang N, Tang YH, Zhang YF, Lee CS, Bello I, Lee ST (1999) Si Nanowires Grown from Silicon Oxide. Chem Phys Lett 299(2):237-242

Wang N, Tang YH, Zhang YF, Yu DP, Lee CS, Bello I, Lee ST (1998) Transmission electron microscopy evidence of the defect structure in Si nanowires synthesized by laser ablation. Chem Phys Lett 283:368-372

Wang Y, Schmidt V, Senz S, Go"sele U (2006) Epitaxial growth of silicon nanowires using an aluminium catalyst. Nat Nanotechnol 1:186-189 
Werner P, Zakharov ND, Gerth G, Schubert L, Go"sele U (2006) On the formation of Si nanowires by molecular beam epitaxy. Int J Mater Res 97:1008-1015

Yang YH, Wu SJ, Chiu HS, Lin PI, Chen YT (2004) Catalytic Growth of Silicon Nanowires Assisted by Laser Ablation. J Phys Chem B 108(3):846-852

Zakharov ND, Werner P, Gerth G, Schubert L, Sokolov L, Go"sele U (2006) Growth phenomena of $\mathrm{Si}$ and $\mathrm{Si} / \mathrm{Ge}$ nanowires on $\mathrm{Si}\left(\begin{array}{lll}1 & 1 & 1\end{array}\right)$ by molecular beam epitaxy. J Cryst Growth 290(1):6-10

Zhang YF, Tang YH, Lam C, Wang N, Lee CS, Bello I, Lee ST (2000) Bulk-quantity Si nanowires synthesized by SiO sublimation. J Cryst Growth 212:115-118

Zhang YF, Tang YH, Peng HY, Wang N, Lee CS, Bello I, Lees ST (1999) Diameter modification of silicon nanowires by ambient gas. Appl Phys Lett 75:1842-1844

Zhang YF, Tang YH, Wang N, Yu DP, Lee CS, Bello I, Lee ST (1998) Silicon nanowires prepared by laser ablation at high temperature. Appl Phys Lett 72:1835-1837

Zheng Y, Rivas C, Lake R, Alam KK, Timothy BB, Gerhard K (2005) Electronic Properties of Silicon Nanowires. Electron Devices, IEEE Transactions on 52 (6):1097-1103

Zhou GW, Zhang Z, Bai ZG, Feng SQ, Yu DP (1998) Transmission electron microscopy study of Si nanowires. Appl Phys Lett 73:677-679

Zhu J, Yu Z, Burkhard GF, Hsu CM, Connor ST, Xu Y, Wang Q, McGehee M, Fan S, Cui Y (2009) Optical Absorption Enhancement in Amorphous Silicon Nanowire and Nanocone Arrays. Nano Lett 9(1):279-282

doi:10.1186/2193-1801-2-151

Cite this article as: Hasan et al: A review on electronic and optical properties of silicon nanowire and its different growth techniques. SpringerPlus 2013 2:151.

\section{Submit your manuscript to a SpringerOpen ${ }^{\circ}$ journal and benefit from:}

- Convenient online submission

- Rigorous peer review

- Immediate publication on acceptance

- Open access: articles freely available online

- High visibility within the field

- Retaining the copyright to your article 